\title{
Propiedades Antropométricas y Somatotipo de Jugadores de Baloncesto de Diferente Nivel Competitivo
}

\author{
Anthropometric Properties and Somatotype of Basketball Players from Different Competition Level
}

\author{
Juan Manuel Rivera-Sosa ${ }^{* * * * * * *}$
}

RIVERA-SOSA, J. M. Propiedades antropométricas y somatotipo de jugadores de baloncesto de diferente nivel competitivo. Int. J. Morphol., 34(1):179-188, 2016.

RESUMEN: El objetivo del estudio es determinar las propiedades antropométricas y el somatotipo, en una muestra de jugadores de baloncesto varonil de las selecciones del estado de Chihuahua México de diferente nivel competitivo. Se evaluaron 10 variables antropométricas utilizando la técnica ISAK en 64 jugadores de baloncesto, pertenecientes a las selecciones varoniles de Chihuahua México que participaron en los eventos nacionales del 2007 categorías: Infantil, Pasarela, Cadetes, Elite sub-18 y Universitario. Se determinó el somatotipo según el método antropométrico decimal de Carter \& Heath y se comparó con una referencia elite. Resultados: el somatotipo del jugador de baloncesto de diferente nivel competitivo de Chihuahua México es Mesomorfo - Ectomórfico con valor medio de 2,38-4,66-3,21 y sin embargo, difiere del referente elite internacional con un SAD mayor a una unidad somatotípica (S). La población evaluada presenta un somatotipo similar entre las categorías universitario, elite y cadetes, lo cual sugiere un proceso deportivo concordante a lo esperado a partir de esta categoría; la categoría pasarela se distingue con un físico más lineal que universitarios. Los resultados del presente estudio pueden ser considerados un referente idóneo para nuestra población, considerando las características específicas de las muestras evaluadas y el logro deportivo obtenido por las mismas. Sin embargo el somatotipo de encontrado en cada categoría de competición evaluada, puede ser considerado un referente deportivo para cada categoría para la población mexicana en esta disciplina deportiva, dado el logro deportivo de las muestras evaluadas.

PALABRAS ClAVE. Antropometría; Tamaño Corporal; Somatotipo; Baloncesto; Nivel de Competición.

\section{INTRODUCCIÓN}

El estudio morfológico en atletas, se ha dirigido en un primer momento a detectar y determinar los cambios durante el crecimiento, y los promovidos por el ejercicio. Los cambios físicos tienen especial significado cuando se relacionan a una práctica deportiva sistematizada, y en particular con una especialización deportiva. La evaluación cineantropométrica y en especial el somatotipo, son de utilidad para la selección y el control de los atletas en formación. Los estudios en este ámbito se han enfocado a documentar y analizar las dimensiones: morfológicas, funcionales y de tipo técnico táctico deportivo del atleta, caracterizándolos en función del deporte, posición o prueba (Norton \& Olds, 1996; Popovic et al., 2013).

En el contexto de la evaluación del deportista, las técnicas que en general han sido utilizadas tradicionalmente son la valoración antropométrica y sus diferentes estimaciones que de estos datos se derivan, los test de evaluación de la condi- ción física y aquellos enfocados a las habilidades técnicas o destrezas motoras de cada deporte (Apostolidis et al., 2003).

Es necesario plantear y utilizar procesos de valoración del jugador de baloncesto durante todas las fases de formación deportiva, para documentar los procesos morfológicos, correspondientes a cada una de las etapas determinadas por las diferentes categorías de competición (Carlson et al., 1994; Jecilic et al., 2002; Drinkwater et al., 2008). Orientar la evaluación deportista, tanto para la diferenciación como del reconocimiento de la similitud entre los deportistas, permite identificar la efectividad de los procesos de formación revelar con ello la especificidad y diferenciación deportiva. Lo cual se postula, es adquirida y definida como producto del entrenamiento, tanto por lo específico de su deporte como por el nivel a que pertenece, con influencia indirecta de factores diversos como la nutrición y la base genética de raza y género que el individuo posea.

* Facultad de Ciencias de la Cultura Física, Universidad Autónoma de Chihuahua, Chihuahua, México.

** Laboratorio para la Actividad Física y la Salud, Universidad Autónoma de Chihuahua, Chihuahua, México.

**** Delegado Estatal de FIEP Chihuahua, por La Federación Internacional De Educación Física (Fiep), Delegación México, Chihuahua, México. 
La evaluación cineantropométrica en el entrenamiento deportivo es un recurso poco utilizado en México para apoyar los procesos de formación deportiva. Usualmente suelen ser vistos y realizados bajo premisas empíricas o poco sistemáticas, y en muchos de los casos son considerados innecesarios por los entrenadores. Ante esta perspectiva, la detección de un niño o adolescente que es visto como futuro talento o atleta, solo se presenta si ha figurado como ganador en competencias de diferentes niveles, más no, como el producto de un proceso.

Hoare (2000) indica que al analizar datos antropométricos en jóvenes basquetbolistas considerados como mejores jugadores, difieren del resto para ambos géneros. Por lo cual, uno de los aspectos básicos a evaluar, es la forma y composición de su cuerpo por medio del método de valoración del somatotipo de Carter \& Heath (1990). El somatotipo se define como la cuantificación de la forma y composición corporal presente del individuo y expresado por tres componentes relativos a la estatura: endomorfia, mesomorfia y ectomorfia. Donde el componente endomorfia determina la grasa relativa a la estatura (adiposidad); la mesomorfia indica el desarrollo músculo esquelético relativo a la estatura (robustez); y la ectomorfia representa la linealidad corporal (delgadez), lo cual se representa por medio de sus tres numerales o como un punto dentro de un gráfico especifico nombrado somatocarta. El somatotipo es uno de los recursos más confiables para la cuantificación y representación grafica de la forma física y composición corporal de una persona. Sus propiedades metodológicas permiten valorar tanto el físico de niños y adultos, haciendo posible la comparación entre cualquier tipo de poblaciones o individuos (Carter \& Heath; Norton \& Olds, 1996).

El método del somatotipo antropométrico decimal de Carter \& Heath ha sido muy útil para describir las variaciones del físico en los periodos puberal, durante la adolescencia y en estudios longitudinales, documentando los patrones relativamente constantes durante el periodo de crecimiento, con lo cual se ha identificado las etapas de marcada fluctuación en la dimensión corporal (Veldre, 2004).

El somatotipo del jugador de Baloncesto varonil, ha sido documentado en varios estudios en diferentes poblaciones (Toriola et al., 1985; Carter \& Heath; Rivera \& Suarez, 1990; Viviani, 1994; Norton \& Olds, 1996; Jecilic et al., 2002; Rivera-Sosa, 2006; Ochoa et al. 2014; Valdez-Badilla, et al. 2015) indicando en la mayoría de ellos para los hombres un somatotipo mesomorfo-ectomorficos, mesomorfoectomorfos y ectomorfo-mesomorficos. Costa (2005) reporta un somatotipo medio para el baloncesto de 2,37-4-07-3,46 (mesomorfo-ectomorfico), derivado de selecciones olímpicas (1976) y para diferentes selecciones nacionales de ba- loncesto (México, Australia, Rusia, Cuba, España, Italia y Sudáfrica) entre 1984 y 1998. El somatotipo reportado para la selección olímpica mexicana de 1984, 2,2-4,3-3,5 (mesomorfo-ectomorfico) en consistencia con lo planteado por Carter \& Heath. Sin embargo, la cantidad de publicaciones que abordan el baloncesto es considerablemente menor, respecto a otras disciplinas deportivas de conjunto (Drinkwater et al.), lo cual le da vigencia y pertinencia al presente estudio.

El objetivo del presente estudio fue determinar las propiedades antropométricas de base para el tamaño corporal y el somatotipo, en una muestra de jugadores de baloncesto varonil de las selecciones de Chihuahua México de diferente nivel competitivo.

\section{MATERIAL Y MÉTODO}

El presente estudio es de tipo transversal, descriptivo y comparativo, no probabilístico. Se evaluaron 5 selecciones varoniles de baloncesto:Infantil, Pasarela, Cadete, Elite sub-18 y Universitaria. Cada selectivo estuvo conformado por hasta 14 sujetos y un mínimo de 12 . Todos los entrenadores, padres de familia o tutores y atletas, fueron informados previamente sobre las condiciones y propiedades de la evaluación, a lo cual accedieron voluntariamente y firmaron un consentimiento informado.

Las sesiones de evaluación se establecieron bajo programación previa con los entrenadores, y se realizaron en los gimnasios donde realizaban sus entrenamientos cuatro semanas antes de su competencia nacional.

La evaluación antropométrica se realizó por duplicado considerando el perfil completo de acuerdo a la técnica de la International Society for Advancement of Kinanthropometry (ISAK, 2006), realizada por un antropometrista certificado ISAK Nivel 3. Sin embargo, solo se reportan las 10 variables necesarias al somatotipo, mas estatura sentado y envergadura (Ross \& Marfell-Jones, 2005).

Se utilizó un Kit Antropométrico Centurión marca Rosscraft $^{\circledR}$ (Innovations Anthropometric Devices): dos calibradores Slimguide ${ }^{\circledR}$ para medición de pliegues cutáneos, dos cintas antropométricas metálicas $0,008 \mathrm{~m}$ de ancho marca Lufkin $^{\circledR}$, dos calibradores Campbell ${ }^{\circledast}$ modelo corto y largo (con extensores de profundidad), un segmómetro ${ }^{\circledR}$, un estadímetro de pared, todos con lectura mínima de $0,001 \mathrm{~m}$ y un banco de madera nivelado con medidas 40 x 40 x 40 $\mathrm{cm}$. El registro de masa corporal se obtuvo con una bascula 
portátil marca Tanita ${ }^{\circledR}$ con un registro mínimo de $0,001 \mathrm{~kg}$.

El error técnico de medición relativo (\%) en presente estudio, fue menor a los estándares establecidos para el antropometrista nivel 3, según los criterios de la ISAK (Tabla I).

La superficie corporal (SC) se determinó utilizando la ecuación indicada por Ross \& Kerr (1993): SC = Csc *

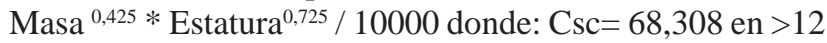
años; 70,619 en $<12$ años.

Se determino el somatotipo antropométrico por el método ecuacional de Carter \& Heath, el cual requiere: masa corporal, estatura, los pliegues cutáneos de triceps, subescapular, supraespinale y pierna medial, las circunferencias de brazo tensionado y pierna máxima, y los diámetros óseos de humero y fémur. Se obtuvo también la distancia posicional tridimensional somatotípica (Somatotype Atitudinal Distance) y la distancia posicional media (Somatotype Atitudinal Media), SAD y SAM respectivamente por sus siglas en inglés. Ambos valores son utilizadas como criterios de medida para la diferenciación y dispersión somatotípica (Carter, 2002).

Para el registro de la fotografía somatotípica, se utilizó al final de la evaluación antropométrica, una cámara digital marca Sony Cyber-shot modelo DSC-P73, con lente Sony (optical 3X, $f=6-18 \mathrm{~mm}$ 1:2,8-5,2) la cual estuvo empotrada en un tripié mini-D con altura graduable, colocada a la altura del centro del cuerpo. Se utilizo un disco giratorio marca Rosscraft ${ }^{\circledR}$ donde se coloco el sujeto de pie, manteniendo la postura correcta frente a la cámara (anterior, lateral izquierdo y posterior) sin necesidad de cambiar el apoyo de sus pies.

El análisis estadístico se realizo en SPSS versión 15.0 para Windows ${ }^{\circledR}$. El test de Kolmogorov-Smirnof para muestras menores de 30 casos se utilizo para el análisis de normalidad. Dado que los datos presentaron comportamientos normales y no normales, fue necesario aplicar ambas técnicas paramétricas (p) y no paramétricas (np). Se utilizaron las pruebas ANOVA con post hoc de Sheffé y la prueba U de Mann-Whitney, con un nivel de confianza estadístico de
$95 \%$ de confiabilidad y un a= 0,05 considerando una comparación general y por pares respecto a su categoría inmediata inferior.

\section{RESULTADOS}

En la Tabla I se indican los resultados promedio del error técnico de medición para las variables antropométricas evaluadas, el cual fue menor al límite establecido por la ISAK: en pliegues cutáneos, menor al $5 \%$ y el resto de las variables menor al $1 \%$. Estos resultados, permite establecer la confiabilidad de todos los valores antropométricos obtenidos y sus respectivas estimaciones.

Propiedades antropométricas. En la Tabla II se indican las Medias $\pm D E$ de la edad de cada categoría de competición, así como el lugar obtenido por los selectivos de Chihuahua México en los eventos nacionales del 2007 para cada categoría. Por el lugar obtenido en su competencia nacional, se considera a la muestra de sujetos, representativa del jugador de baloncesto de mayor nivel para México al momento de la evaluación.

En el presente estudio las variables evaluadas que determinan el tamaño corporal del jugador de baloncesto son: la masa corporal, la estatura, estatura sentado, envergadura y superficie corporal, las cuales se presentan en la Tabla II. La edad y el tamaño corporal son dos aspectos que mostraron diferencias significativas y que explican el tamaño corporal entre las muestras evaluadas $(\mathrm{P} \leq 0,001)$ y entre todas las comparaciones subsecuentes $(\mathrm{P} \leq 0,001)$. En relación al análisis de la masa corporal, el ANOVA indicó que los jugadores de la categoría universitaria presentaron una masa corporal similar para elite y cadetes. Mientras que aquellos con menor masa corporal respecto a la categoría universitaria fueron las categorías pasarela e infantil $(\mathrm{P}=0,000)$. La categoría pasarela por otro lado, fue la única que presentó mayor masa corporal que la categoría subsecuente infantil $(\mathrm{P}=0,000)$. El análisis de los resultados de la estatura (prueba U Mann-Whitney), indican que las categorías en formación elite y cadetes son similares a la universitaria, mientras que las categorías pasarela e infantil son menores $(\mathrm{P}=0,000)$.

Tabla I. Error técnico de medición (TEM) de las variables antropométricas evaluadas y su correspondiente Índice de Correlación Intraclase (ICC).

\begin{tabular}{lccc}
\hline Variables & $\begin{array}{c}\text { TEM Absoluto (mm) } \\
\text { Media \#DE }\end{array}$ & $\begin{array}{c}\text { TEM Relativo } \\
\text { Media \#DE }\end{array}$ & $\begin{array}{c}\text { ICC } \\
\text { Media \#DE }\end{array}$ \\
\hline Pliegues & $0,32 \pm 0,04$ & $3,74 \pm 0,54$ & $0,98 \pm 0,01$ \\
Circunferencias & $0,22 \pm 0,11$ & $0,55 \pm 0,23$ & $0,98 \pm 0,03$ \\
Diámetros & $0,14 \pm 0,14$ & $0,72 \pm 0,19$ & $0,03 \pm 0,03$ \\
\hline
\end{tabular}

Donde: ICC= Coeficiente de Correlación Intraclase; DE= Desviación Estándar. 
Tabla II. Edad decimal y tamaño corporal de jugadores de baloncesto en función de categorías de competición (Media \pm Desviación Estándar).

\begin{tabular}{|c|c|c|c|c|c|}
\hline Categoría & $\begin{array}{c}\text { Universitario } \\
(n=14)\end{array}$ & $\begin{array}{c}\text { Elite } \\
(n=14)\end{array}$ & $\begin{array}{l}\text { Cadete } \\
(n=13)\end{array}$ & $\begin{array}{c}\text { Pasarela } \\
(\mathrm{n}=12)\end{array}$ & $\begin{array}{r}\text { Infantil } \\
(n=12)\end{array}$ \\
\hline Ranking Nacional (2007) & $3^{\text {er }}$ Lugar & $2^{\circ}$ Lugar & $1^{\text {er }}$ Lugar & $1^{\text {er } L_{u g a r}}$ & $1^{\text {er }} \mathrm{L}_{\text {ugar }}$ \\
\hline Edad decimal (años) ${ }^{p}$ & $\begin{array}{l}20,4^{*} \pm 1,67 \\
(P=0,000)\end{array}$ & $\begin{array}{l}17,23 u^{*} \pm 0,61 \\
(P=0,000, P=0,000)\end{array}$ & $\begin{array}{l}15,45 \mathrm{u} * \pm 0,58 \\
(P=0,000, P=0,001)\end{array}$ & $\begin{array}{l}13,80^{\mathrm{u}} * \pm 0,47 \\
(P=0,000 ; P=0,000)\end{array}$ & $\begin{array}{l}11,90^{\mathrm{u}} \pm 0,17 \\
(P=0,000)\end{array}$ \\
\hline $\operatorname{Masa}(\mathbf{k g})^{p}$ & $\begin{array}{l}88,08 \pm 17,38 \\
---\end{array}$ & $\begin{array}{l}83,14 \pm 13,47 \\
---\end{array}$ & $\begin{array}{l}74,26 \pm 7,78 \\
---\end{array}$ & $\begin{array}{l}63,65^{\mathrm{u}} * \pm 6,88 \\
(P=0,000 ; P=0,000)\end{array}$ & $\begin{array}{l}47,87^{\mathrm{u}} \pm 10,04 \\
(P=0,000)\end{array}$ \\
\hline Estatura (cm) ${ }^{n p}$ & $\begin{array}{l}187,66 \pm 9,21 \\
---\end{array}$ & $\begin{array}{l}185,31 \pm 7,28 \\
---\end{array}$ & $\begin{array}{l}183,57 * \pm 6,65 \\
(P=0,003)\end{array}$ & $\begin{array}{l}176,94^{4} * \pm 6,9 \\
(P=0,000 ; P=0,000)\end{array}$ & $\begin{array}{l}158,79^{\mathrm{u}} \pm 13,01 \\
(P=0,000)\end{array}$ \\
\hline Estatura sentado $(\mathrm{cm}) n p$ & $\begin{array}{l}97,82 \pm 5,04 \\
---\end{array}$ & $\begin{array}{l}96,95 \pm 4,77 \\
(\mathrm{n}=13)\end{array}$ & $\begin{array}{l}94,74 u * \pm 2,87 \\
(P=0,043 ; P=0,01)\end{array}$ & $\begin{array}{l}91,08^{u} * \pm 2,95 \\
(P=0,000 ; P=0,006)\end{array}$ & $\begin{array}{l}83,11^{\mathrm{u}} \pm 6,86 \\
(P=0,000)\end{array}$ \\
\hline Envergadura $(\mathbf{c m}) n p$ & $\begin{array}{l}194,93 \pm 10,13 \\
---\end{array}$ & $\begin{array}{l}194,06 \pm 6,57 \\
(\mathrm{n}=13)\end{array}$ & $\begin{array}{l}188,23^{*} \pm 9,18 \\
(P=0,046)\end{array}$ & $\begin{array}{l}181,10^{4} * \pm 7,22 \\
(P=0,000 ; P=0,001)\end{array}$ & $\begin{array}{l}159,49^{\mathrm{u}} \pm 19,33 \\
(P=, 000)\end{array}$ \\
\hline Superficie Corporal $\left(\mathbf{m}^{2}\right) p$ & $\begin{array}{l}2,03 \pm 0,23 \\
---\end{array}$ & $\begin{array}{l}1,97 \pm 0,18 \\
---\end{array}$ & $\begin{array}{l}1,86 \pm 0,11 \\
---\end{array}$ & $\begin{array}{l}1,70^{\mu} * \pm 0,11 \\
(P=0,000 ; P=0,003)\end{array}$ & $\begin{array}{l}1,39^{u} \pm 0,21 \\
(P=0,000)\end{array}$ \\
\hline
\end{tabular}

Donde: $\mathrm{p}=$ Valores Paramétricos; $\mathrm{np}=$ Valores No Paramétricos; Significancia $\mathrm{p} \leq 0,05: \mathrm{u}=$ respecto a universitario; * respecto a la categoría inmediata inferior; las pruebas paramétricos fueron ANOVA y post hoc Sheffé; la prueba no paramétrica fue la prueba U de Mann - Whitney. El Ranking Nacional se determino por el lugar obtenido en las competencias nacionales de su categoría en ese año (2007).

Al compararse cada categoría con su inmediata inferior, cadetes fueron mayores que pasarela $(\mathrm{P}=0,01)$ y pasarela mayores que infantiles $(\mathrm{P}=0,000)$. Los resultados obtenidos para la variable envergadura (tabla II), muestran que universitarios son similares a elite y cadetes. En universitarios es mayor que pasarela e infantil $(\mathrm{P}=0,000)$. Los cadetes fueron mayores en envergadura que pasarela $(\mathrm{P}=0,046)$, mientras que pasarela fue mayor a infantil $(\mathrm{P}=0,001)$. El tamaño del tronco valorado desde la estatura sentada del deportista, presentó diferencias significativas principalmen- te a partir de las categorías menores antes de los 14 años donde la proporción del tronco es menor para los jugadores de baloncesto aquí evaluados.

En superficie corporal $\left(\mathrm{SC}^{2}\right)$ los jugadores universitarios, elite y cadetes no presentan diferencias significativas entre sí, mientras que universitarios es mayor que pasarela e infantil $(\mathrm{P}=0,000)$. En el comparativo por pares $(*)$, solo pasarela es mayor en superficie corporal que infantil $(\mathrm{P}<0,001)$.

Tabla III. Propiedades Antropométricas (Media \pm Desviación Estándar) de jugadores de baloncesto en función de categorías de competición.

\begin{tabular}{|c|c|c|c|c|c|c|}
\hline Variables & & $\begin{array}{l}\text { Universitario } \\
\quad(n=14)\end{array}$ & $\begin{array}{c}\text { Elite } \\
(n=14)\end{array}$ & $\begin{array}{l}\text { Cadete } \\
(n=13)\end{array}$ & $\begin{array}{c}\text { Pasarela } \\
(n=12)\end{array}$ & $\begin{array}{l}\text { Infantil } \\
(n=12)\end{array}$ \\
\hline \multirow[t]{8}{*}{ Pliegues Cutáneos } & Triceps $(\mathrm{mm})^{n p}$ & $8,14 \pm 2,97$ & $8,87 \pm 2,75$ & $8,65 \pm 3,37$ & $7,46 * \pm 2,56$ & $10,04 \pm 2,46$ \\
\hline & & --- & --- & --- & $(\mathrm{P}=0,014)$ & --- \\
\hline & Subescapular (mm) $n p$ & $10,73 \pm 4,53$ & $9,48 \pm 2,73$ & $8,87 * \pm 2,40$ & $6,58^{\mathrm{u}} \pm 1,21$ & $6,85^{\mathrm{u}} \pm 1,00$ \\
\hline & & --- & --- & $(\mathrm{P}=0,003)$ & $(\mathrm{P}=0,000)$ & $(\mathrm{P}=0,000)$ \\
\hline & Supraespinale (mm) ${ }^{n p}$ & $9,45 \pm 5,45$ & $9,56 \pm 4,92$ & $8,63 \pm 5,84$ & $6,02 * \pm 2,15$ & $7,56 \pm 2,16$ \\
\hline & & --- & --- & --- & $(\mathrm{P}=0,045)$ & --- \\
\hline & 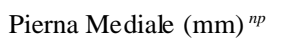 & $6,30 \pm 2,65$ & $7,12+2,68$ & $7,85 \pm 2,66$ & $7,00 * \pm 2,00$ & $10,1^{\mathrm{u}} \pm 2,92$ \\
\hline & & --- & --- & --- & $(\mathrm{P}=0,006)$ & $(\mathrm{P}=0,003)$ \\
\hline \multirow[t]{3}{*}{ Circunferencias } & Brazo Tensionado $(\mathrm{cm})^{p}$ & $34,4 \pm 3,06$ & $32,9 \pm 2,55$ & $\begin{array}{c}30,97^{\mathrm{u}} \pm 2,09 \\
(\mathrm{P}=0.015)\end{array}$ & $\begin{array}{c}28,5^{\mathrm{u} *} \pm 1,67 \\
(\mathrm{P}=0,000 \cdot \mathrm{p}=0,022)\end{array}$ & $\begin{array}{c}24,9^{\mathrm{u}} \pm 2,42 \\
(\mathrm{P}=0,000)\end{array}$ \\
\hline & Pierna Máxima $(\mathrm{cm})^{p}$ & $39,14 \pm 2,91$ & $38,50 \pm 2,38$ & $35,95^{\mathrm{u}} \pm 1,87$ & $35,4^{\mathrm{u} * \pm} 1,84$ & $31,7^{\mathrm{u}} \pm 2,83$ \\
\hline & & --- & --- & $(\mathrm{P}=0,028)$ & $(\mathrm{P}=0,007 ; \mathrm{p}=0,012)$ & $(\mathrm{P}=0,000)$ \\
\hline \multirow[t]{4}{*}{ Diá metros Óseos } & Húmero $(\mathrm{cm})^{n p}$ & $7,37 \pm 0,33$ & $7,53 \pm 0,37$ & $7,43 \pm 0,25$ & $7,25 * \pm 0,24$ & 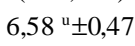 \\
\hline & & --- & --- & --- & $(\mathrm{P}=0,000)$ & $(\mathrm{P}=0,004)$ \\
\hline & Fémur $(\mathrm{cm})^{n p}$ & $10,34 \pm 0,52$ & $10,32 \pm 0,41$ & $10,32 \pm 0,19$ & $10,2 * \pm 0,33$ & $9,56^{\mathrm{u}} \pm 0,59$ \\
\hline & & --- & --- & --- & $(\mathrm{P}=0,000)$ & $(\mathrm{P}=0,000)$ \\
\hline
\end{tabular}

Donde: $\mathrm{n}=$ Valores Paramétricos; $\mathrm{np}=$ Valores No Paramétricos; Significancia $\mathrm{P} \leq 0,05$ : $\mathrm{u}=$ respecto a universitario; $*$ respecto a la categoría inmediata inferior; la prueba paramétrica fue ANOVA y el post hoc Sheffé; la prueba no paramétrica fue U de Mann-Whitney. 
Los resultados de adiposidad en pliegues cutáneos se indican en la Tabla III (A), muestra que universitarios presentaron una adiposidad similar a elite y cadetes en todos los sitios. Sin embargo, universitarios respecto a infantil y pasarela fue mayor en el pliegue subescapular $(\mathrm{P}=0,000)$, pero infantiles es mayor que universitarios en pierna medial $(\mathrm{P}=0,003)$. La comparación por pares $(*)$, mostró diferencias únicamente entre cadetes y pasarela en subescapular $(\mathrm{P}=0,003)$, y entre pasarela e infantil en los pliegues de triceps $(\mathrm{P}=0,014)$, supraespinale $(\mathrm{P}=0,045)$, y pierna medial $(\mathrm{P}=0,006)$, siendo menores en todos los casos en pasarela.

La amplitud de la sección transversal a partir de las circunferencias evaluadas, se presenta en la Tabla III (B). La comparación de universitarios respecto a las categorías en formación, se encontraron diferencias respecto a cadetes, pasarela e infantiles $(\mathrm{P}<0,05)$ siendo mayores en universitarios, no así respecto a elite ante quienes no se encontraron diferencias. En las comparaciones subsecuentes, solo pasarela fue mayor que infantiles en ambas circunferencias $(\mathrm{P} \leq 0,03)$.

En diámetros óseos, los universitarios en estas variables no presentaron diferencias respecto de elite, cadetes y pasarela, indicando una similitud en humero y fémur, ver Tabla III (C). Por otro lado, infantil presentó diferencias respecto a universitarios, en ambos diámetros óseos con menor dimensión $(\mathrm{P}<0,01)$; nuevamente en las comparaciones por pares $(*)$, presentaron diferencias en ambos diámetros únicamente entre pasarela respecto a infantiles, siendo mayores en pasarela $(\mathrm{P}<0,000)$.
Somatotipo. La Tabla IV refiere los valores medios del somatotipo y su desviación estándar por componente para cada muestra evaluada y una media general $(n=64)$. El análisis descriptivo indica predominancia de la mesomorfia, con valores moderados entre 4,5 y 5 . En endomorfia se presentaron valores bajos entre 1,83 y 2,66 donde infantil obtuvo un valor con mayor magnitud $(2,66)$ y pasarela presento el valor más bajo $(1,83)$. Por otro lado, en ectomorfia solo los valores de universitario y elite son considerados bajos $(2,46$ y 2,65 respectivamente), mientras que para el resto se consideran moderados con valores entre 3,44 y 3,92 para este componente. El análisis comparativo mostro en índice ponderal (HWR) diferencias entre universitarios y pasarela siendo mayor en los últimos $(\mathrm{P}=0,033)$ lo cual explica las diferencias en endomorfia mayor en universitarios $(\mathrm{P}=0,014)$ y la mayor ectomorfia en pasarela $(\mathrm{P}=0,033)$. Es notorio que no se encontraron diferencias significativas entre ninguna de las comparaciones para el componente mesomorfico, lo cual sugiere un físico relativamente similar para toda la muestra evaluada. Al determinar la categoría somatotípica de cada muestra, universitario y elite corresponden a la categoría mesomorfo balanceado, mientras que cadete, pasarela e infantil corresponden a la categoría mesomorfo ectomorfico. El somatotipo promedio general para este estudio es 2,38-4,66-3,21 (DE 0,32-0,3-0,62) el cual corresponde a la categoría mesomorfo ectomorfico para a la totalidad de la muestra evaluada $(n=64)$ para el jugador de baloncesto considerado ganador. El valor de la distancia posicional somatotípica respecto al referente elite fue menor únicamente para infantil, quienes fueron más cercanos al referente $(\mathrm{SAD}=1,07 \mathrm{~S}, \mathrm{P}=0,004)$; mientras que en $\mathrm{SAM}$

Tabla IV. Somatotipo General y comparación por categoría de competición de jugadores de baloncesto de Chihuahua México (Media \pm Desviación Estándar).

\begin{tabular}{|c|c|c|c|c|c|c|}
\hline & $\begin{array}{c}\text { General } \\
(n=64)\end{array}$ & $\begin{array}{l}\text { Universitario } \\
\quad(n=14)\end{array}$ & $\begin{array}{c}\text { Elite } \\
(n=14)\end{array}$ & $\begin{array}{l}\text { Cadete } \\
(n=13)\end{array}$ & $\begin{array}{c}\text { Pasarela } \\
(n=12)\end{array}$ & $\begin{array}{c}\text { Infantil } \\
(n=12)\end{array}$ \\
\hline \multirow[t]{2}{*}{ HWR $p$} & $43,38 \pm 1,66$ & $42,39 \pm 1,57$ & $42,65 \pm 1,34$ & $43,75 \pm 1,74$ & $44,4 \pm 1,76$ u & $43,9 \pm 1,01$ \\
\hline & --- & --- & --- & --- & $(\mathrm{P}=0,033)$ & --- \\
\hline \multirow[t]{2}{*}{ Endomorfia $^{n p}$} & $2,38 \pm 0,32$ & $2,51 \pm 1,04$ & $2,53 \pm 0,82$ & $2,37 \pm 1,12$ & $1,83 \pm, 65 *$ & $2,66 \pm 0,68$ \\
\hline & --- & --- & --- & --- & $(\mathrm{P}=0,014)$ & --- \\
\hline Mesomorfia $^{p}$ & $4,66 \pm 0,3$ & $4,97 \pm 0,94$ & $5,00 \pm 0,78$ & $4,35 \pm 1,05$ & $4,45 \pm 0,92$ & $4,54 \pm 0,66$ \\
\hline \multirow[t]{2}{*}{ Ectomorfia $^{p}$} & $3,21 \pm 0,62$ & $2,46 \pm 1,14$ & $2,65 \pm 0,96$ & $3,44 \pm 1,27$ & $3,92 \pm 1,29$ u & $3,56 \pm 0,74$ \\
\hline & --- & --- & --- & --- & $(\mathrm{P}=0,033)$ & --- \\
\hline Categoría & Mesomorfo & Mesomorfo & Mesomorfo & Mesomorfo & Mesomorfo & Mesomorfo \\
\hline Somatotipica & Ectomorfico & Bal anceado & Balanceao & Ectomórfico & Ectomórfico & Ectomórfico \\
\hline \multirow[t]{2}{*}{$\mathrm{SAD}_{n p}$} & $1,6 \pm 0,4$ & $2,09 \pm 0,94$ & $1,62 \pm 0,98$ & $1,69 \pm 0,96$ & $1,57 \pm 0,8$ & $1,07 \pm 0,56^{\mathrm{u}}$ \\
\hline & --- & --- & -- & -- & --- & $(\mathrm{P}=0,004)$ \\
\hline SAM & 0,65 & 1,60 & 1,20 & 1,69 & 1,42 & 1,06 \\
\hline
\end{tabular}

Donde: HWR= Indice Ponderal (estatura / raíz cubica [masa corporal]); SAD= Distancia posicional respecto a somatotipo elite; SAM= Distancia posicional respecto a somatotipo medio (dispersión intragrupo); $\mathrm{p}=$ Valores Paramétricos; $\mathrm{np}=$ Valores No Paramétricos; Significancia P $\leq 0,05$ : $\mathrm{u}=$ respecto a universitario; * = respecto a la categoría inmediata inferior; la prueba paramétrica ANOVA y post hoc Sheffé; la prueba no paramétrico fue U de Mann-Whitney. 
(distancia posicional media intragrupo) es indicador de la dispersión al interior de cada grupo de somatotipos (respecto de su propia media) indica un valor entre 1,06 y 1,6 unidades $\mathrm{S}$ para las muestras evaluadas y de 0,65 unidades $\mathrm{S}$ para el promedio general.

En las Figuras 1 a 5 se muestra la distribución de somatotipos por categoría de competición. Se indica en cada una de las figuras el somatotipo medio (desviación estándar por componente) y la ubicación respecto al somatotipo de referencia elite sugerido por Norton \& Olds (1996): 2,4-4,4-3,7 (mesomorfo ectomórfico), el cual es consistente con lo reportado por Costa para diferentes selecciones nacionales y olímpicas: $2,37-4-07-3,46$ (mesomorfo ectomórfico). En la Figura 6 se muestra en la somatocarta la ubicación de las medias por categoría evaluada, así como el somatotipo general del estudio actual y los referentes considerados.

En la Figura 7 se ejemplifica el somatotipo fotográfico de un jugador de cada categoría evaluada, con lo cual se hace tangible las propiedades morfológicas y el físico presente en los jugadores de baloncesto de lo que podría describirse como el físico de un conjunto de atletas especialistas de baloncesto de diferente nivel competitivo en México. Se reconoce en ello, el potencial de referencia que representa poseer y evaluar el somatotipo al acompañarse de su fotografía al momento de su evaluación.
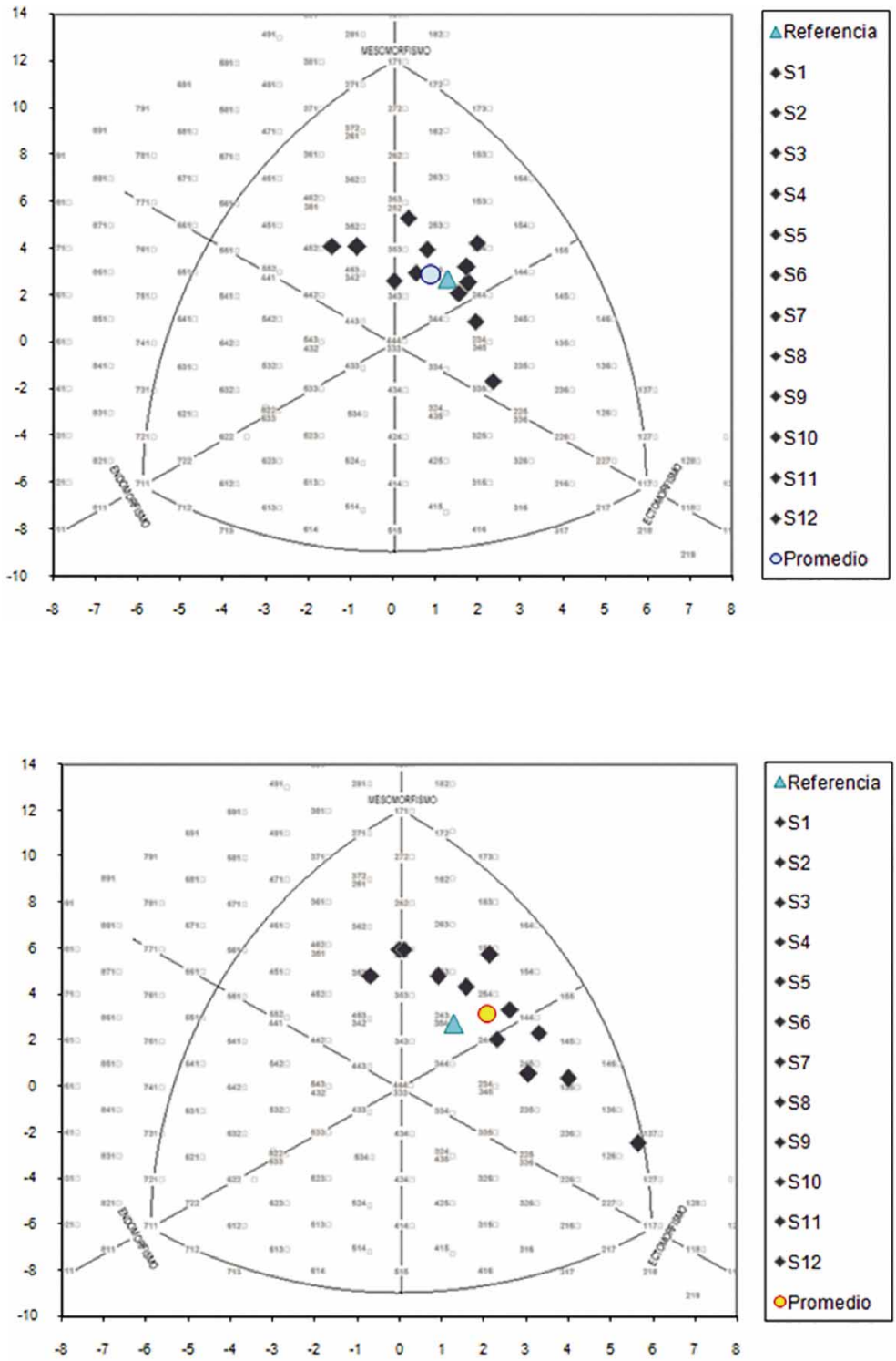

Fig. 1. Distribución de somatotipos de la categoría infantil. Donde el círculo azul indica el somatotipo medio 2,66-4,54-3,56 (0,68; 0,66; $0,74)$ Mesomorfo - Ectomórfico; el triangulo indica el somatotipo de referencia elite 2,4-4,4-3,7; mientras que los rombos negros representan a los jugadores que integran la muestra.
Fig. 2. Distribución de somatotipos de la categoría pasarela. Donde el círculo, indica el somatotipo medio $1,83-4,45-3,92(0,65 ; 0,92 ; 1,29)$ Mesomorfo - Ectomórfico; el triangulo indica el somatotipo de referencia elite 2,4-4,4-3,7; mientras que los rombos negros representan a los jugadores que integran la muestra. 

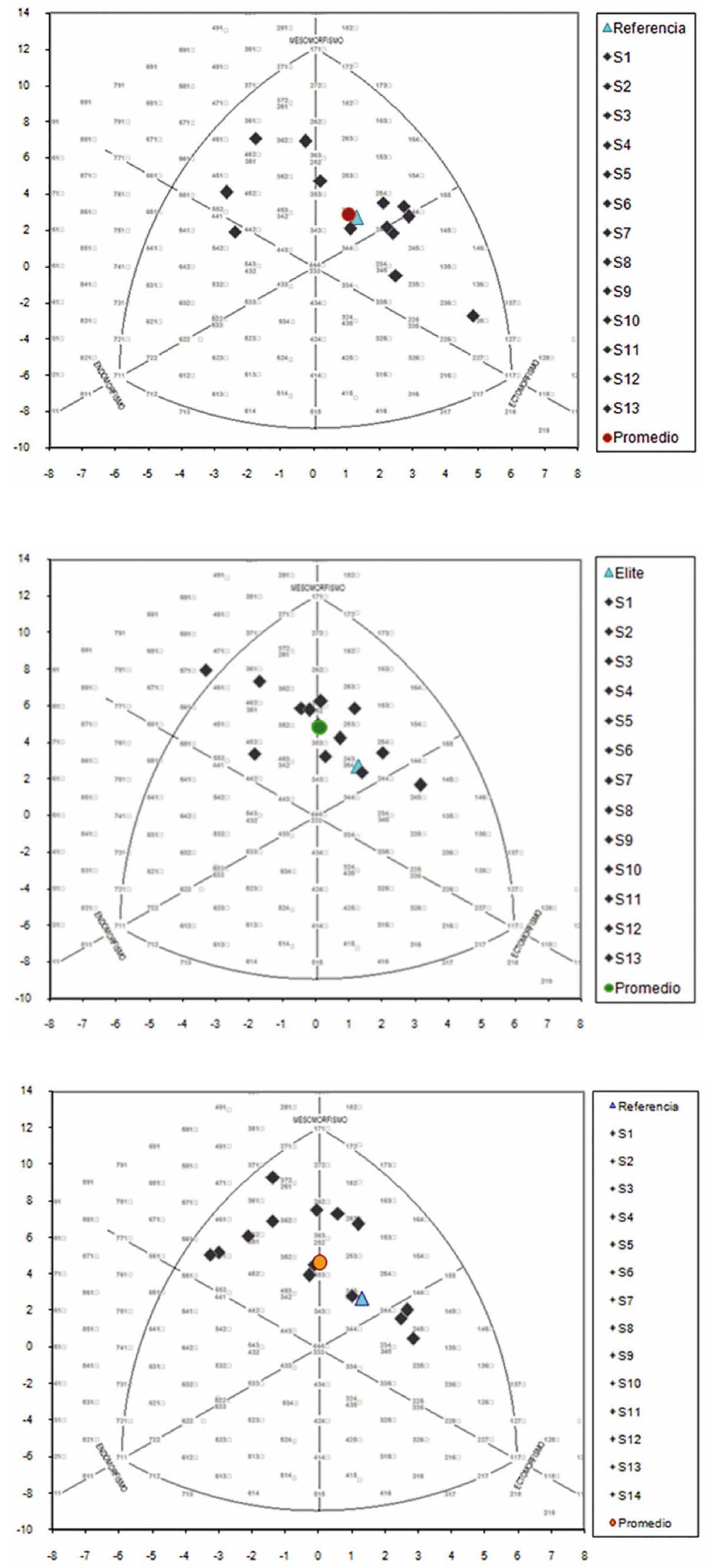

Fig. 3. Distribución de somatotipos de la categoría cadete. Donde el círculo indica el somatotipo medio 2,37-4,35-3,44 (1,12; 1,05; 1,27) Mesomofo - Ectomorfico; el triangulo indica el somatotipo de referencia elite 2,4-4,4-3,7; mientras que los rombos negros representan a los jugadores que integran la muestra.
Fig. 4. Distribución de somatotipos de la categoría elite. Donde el circulo indica el somatotipo medio 2,53-5-2,65 (0,82;0,78;0,96) Mesomorfo - Balanceado; el triangulo indica el somatotipo de referencia elite 2,4-4,4-3,7 mientras que los rombos negros representan a los jugadores que integran la muestra.
Fig. 5. Distribución de somatotipos de la categoría universitaria. Donde el circulo indica el somatotipo medio 2,51-4,97-2,46 (1,04; 0,9; 1,1) Mesomorfo - Balanceado; el triangulo indica el somatotipo de referencia elite 2,4-4,4-3,7 mientras que los rombos negros representan a los jugadores que integran la muestra. 


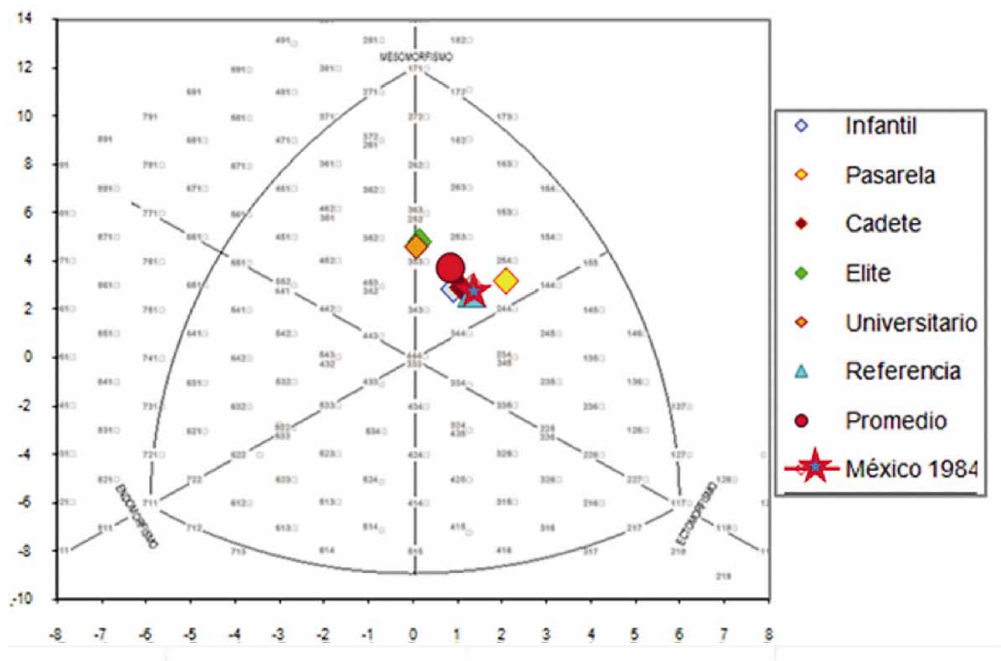

Fig. 6. Distribución del somatotipo promedio de las diferentes categorías de competición (rombos) y el somatotipo Global para el jugador de baloncesto de Chihuahua México de diferente nivel competitivo (círculo rojo) 2,38-4,66-3,21 (Mesomorfo - Ectomórfico); el triangulo (azul cielo) indica el somatotipo de referencia elite 2,4-4,4-3,7 (Norton \& Olds, 1996); mientras que la estrella indica el somatotipo para la selección olímpica mexicana de 1984 con 2,2-4,3-3,5 (Costa, 2005).
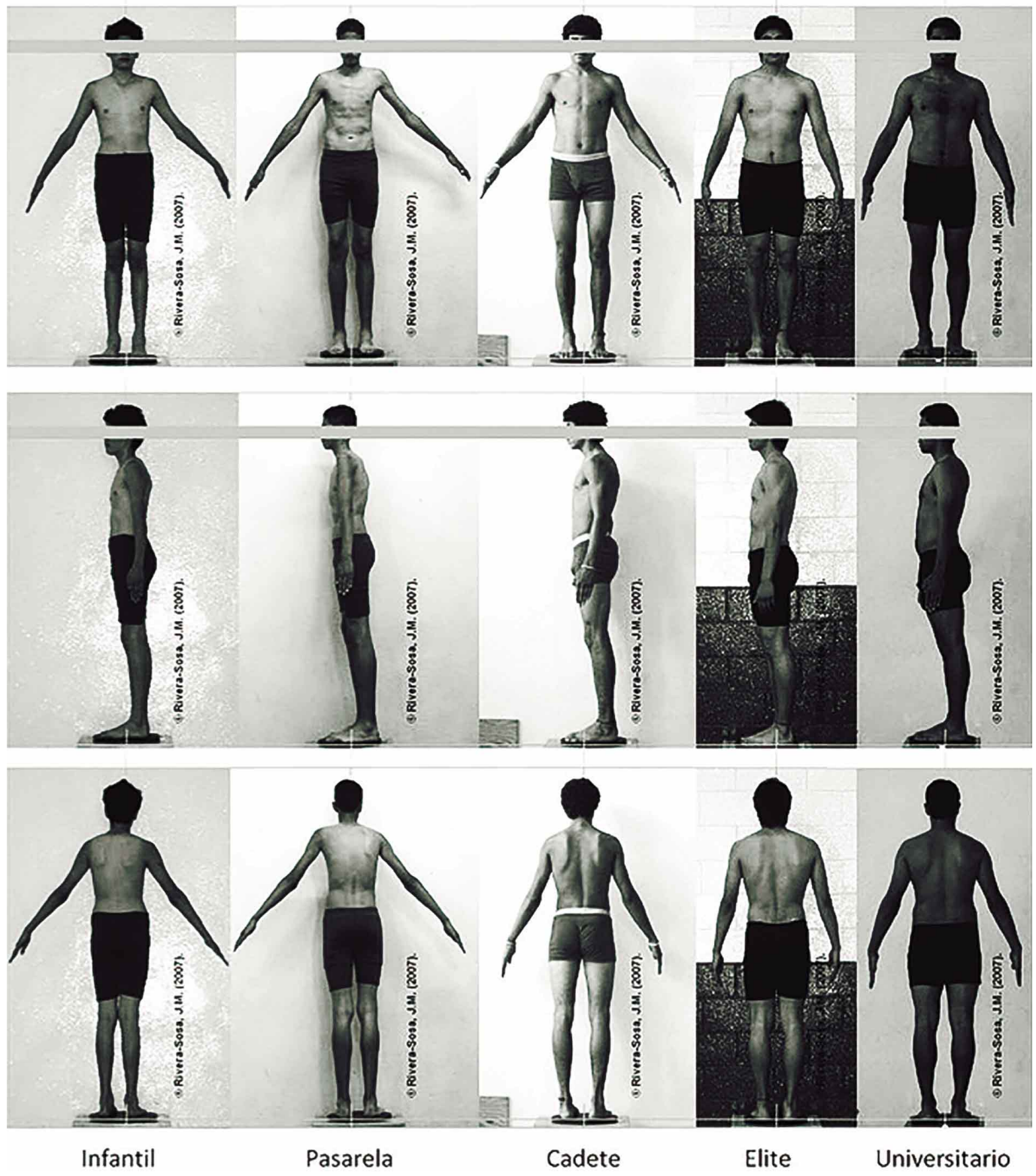

Fig. 7. Ejemplos del físico registrado mediante la fotografía somatotípica del jugador de baloncesto por categoría de competición evaluada (Rivera-Sosa, 2007). 


\section{DISCUSIÓN}

En general los resultados de las variables antropométricas básicas (masa corporal, estatura, estatura sentado y envergadura) muestran junto con la superficie corporal, que las categorías pasarela e infantil son quienes poseen sistemáticamente un menor tamaño corporal que cadetes, elite y universitarios. Sin embargo, cadetes son tendientes a ser similar a elite y universitarios, lo que permite asumir que desde los 15 años el jugador de baloncesto se aproxima al tamaño corporal esperado para la muestra de jugadores de baloncesto considerados ganadores en México. Norton \& Olds (2001) han postulado la tendencia al reclutamiento de jugadores cada vez más altos en el máximo circuito de baloncesto la National Basketball Association, lo cual muestra que la tendencia es el desarrollo de jugadores, no solo con talento sino que siempre será acompañada por un tamaño corporal en franca tendencia a ser cada vez mayor, lo cual es una condición en concordancia a las propiedades de este deporte.

Los pliegues cutáneos son una variable plenamente reconocida como un indicador de la adiposidad y composición del compartimiento de grasa corporal (Norton \& Olds, 1996), con lo cual se reconoce que los valores encontrados en este estudio, fueron menores a lo reportado para el jugador de baloncesto profesional (Ochoa et al.), excepto respecto a infantil en los sitios de tríceps y pierna medial. Sin embargo se reconoce que en los restantes, el valor obtenido es sensiblemente menor para la población del presente estudio que para jugadores profesionales.

Con base en lo anterior, se reconoce una tendencia diferenciada en el somatotipo, donde es palpable una tendencia sistemática a un valor de endomorfia menor para todas las muestras evaluadas en el presente estudio respecto de la muestra de jugadores profesionales mexicanos (Ochoa et al.). Así mismo se reconoce un valor mesomorfico mayor en los profesionales mexicanos por poco más de una unidad somatotípica (S). sin embargo el físico parece más apropiado en las muestras y promedio general del presente estudio respecto de los referentes elite e internacionales al corresponder al criterio de clasificación como un somatotipo mesomorfo ectomorfico (Carter \& Heath), caracterizado por un valor mayor en ectomorfia para nuestro estudio.

Con los datos obtenidos se puede proyectar la utilidad que tiene la evaluación del somatotipo puesto que permite realizar el seguimiento deportivo del atleta de manera permanente. Se determina la similitud en relación al tipo de deporte y se puede reconocer las diferencias propias del nivel deportivo (Rivera-Sosa; Bayios, et al., 2006), con lo cual se reconoce el potencial descriptivo de para monitorizar el proceso de modi- ficación morfológica asociado al entrenamiento (Berdejo et al., 2008) y a la alta especialización deportiva esperado (Carter \& Heath; Green \& Oakley, 2001; Chaouachi et al., 2005; Bayios et al.) la cual se desarrolla como producto de la alta especialización deportiva, adquiriendo cambios físicos que los tipifican dentro de lo esperado para determinada disciplina deportiva y lo distancian de la población promedio (Norton \& Olds, 2001).

Los resultados del presente estudio pueden ser considerados un referente idóneo para nuestra población, considerando las características específicas de las muestras evaluadas y el logro deportivo obtenido por las mismas, tal y como Hoare (2000) lo plantea: comparar "el mejor versus el resto", con lo cual se puede contribuir a los procesos de selección en categorías de competición menores con valores de referencia de nuestra misma población.

CONCLUSIONES. El somatotipo del jugador de baloncesto de diferente nivel competitivo de Chihuahua México es mesomorfo ectomorfico con valor de 2,38-4,66-3,21 (0,32$0,3-0,62)$, aun y cuando pertenece a la misma categoría somatotípica difiere del referente elite internacional. Sin embargo su magnitud por categoría de competición puede ser considerado un referente deportivo para cada categoría para la población mexicana en esta disciplina deportiva.

Los resultados del presente estudio nos muestran que el estudio sistemático del físico del atleta, permite asistir de manera útil el seguimiento y formación deportiva, dada su facilidad y reproducibilidad del estudio antropométrico, con lo cual es posible monitorizar el proceso de modificación morfológica asociado a su práctica deportiva y al perfil morfológico esperado.

AGRADECIMIENTOS. Al Instituto Chihuahuense del Deporte y la Cultura del Estado de Chihuahua México y a la Universidad Autónoma de Chihuahua, instituciones que permitieron el acceso a entrenadores y atletas para el desarrollo del presente estudio. Especial reconocimiento a atletas y padres de familia quienes aceptaron participar en el presente estudio sin los cuales no ese sido posible su realización.

RIVERA-SOSA, J. M. Anthropometric properties and somatotype of basketball players from different competition level. Int. J. Morphol., 34(1):179188, 2016.

SUMMARY: The purpose of this research is to determine the properties and anthropometric somatotype, in a sample of male basketball players selections Mexico state of Chihuahua different competitive level. Child, Catwalk, Cadet, Under-18 and Elite University 10 anthropometric variables using the technique ISAK in 64 basketball players belonging to the masculine selections of Chihuahua Mexico who participated in national events of 2007 
were evaluated categories. Somatotype was determined by the decimal anthropometric method of Carter \& Heath and compared with reference elite. Results: The Somatotype basketball player of different competitive level of Chihuahua Mexico, is ectomorphic mesomorph with mean value of 2.384.66-3.21 and yet differs from elite international benchmark with a larger unit to a somatotype (S) SAD. The study population has a similar somatotype between the university categories elite and cadets, suggesting sporting a matching process expected from this category; category gateway distinguishes with an physical more linear than university the results of this study can be considered an appropriate reference for our population, considering the specific characteristics of the samples tested and athletic achievement obtained by them. However somatotype found in each category of competition evaluated, it can be considered a sports reference for each category for the Mexican population in this sport, given the sporting achievement of the samples tested.

KEY WORDS: Anthropometry; Body size; Somatotype; Basketball; Level of competition.

\section{REFERENCIAS BIBLIOGRÁFICAS}

Apostolidis, N.; Nassis, G. P.; Bolatoglou, T. \& Geladas, N. D. Physiological and technical characteristics of elite young basketball players. J. Sports Med. Phys. Fitness, 44(2):157-63, 2004.

Bayios, I. A.; Bergeles, N. K.; Apostolidis, N. G.; Noutsos, K. S. \& Koskolou, M. D. Anthropometric, body composition and somatotype differences of Greek elite female basketball, volleyball and handball players. J. Sports Med. Phys. Fitness, 46(2):271-80, 2006.

Berdejo del Fresno, D.; Sánchez Pérez, S. \& Jiménez Díaz, F. Composición corporal y carga de entrenamiento en baloncesto: relación directa en el alto nivel. Rev. Digit. (B. Aires), 13(119), 2008.

Carlson, B. R.; Carter, J. E.; Patterson, P.; Petti, K.; Orfanos, S. M. \& Noffal, G. J. Physique and motor performance characteristics of US national rugby players. J. Sports Sci., 12(4):403-12, 1994.

Carter, J. E. L. The Heath-Carter Anthropometric Somatotype. Instruction Manual. San Diego, Department of Exercise and Nutritional Sciences, San Diego State University, 2002.

Carter, J. E. L. \& Heath, B. H. Somatotyping - Development and Applications. Cambridge, Cambridge University Press, 1990.

Chaouachi, M.; Chaouachi, A.; Chamari, K.; Chtara, M.; Feki, Y.; Amri, M. \& Trudeau, F. Effects of dominant somatotype on aerobic capacity trainability. Br. J. Sports Med., 39(12):954-9, 2005.

Costa, I. Características Físico-Fisiológicas de los Jugadores de Basquetbol. PubliCE Standard, 2005. Disponible en: http://g-se.com/es/entrenamiento-en-basquetbol/articulos/caracteristicas-fisico-fisiologicas-de-los-jugadores-de-basquetbol-466

Drinkwater, E. J.; Pyne, D. B. \& McKenna, M. J. Design and interpretation of anthropometric and fitness testing of basketball players. Sports Med., 38(7):565-78, 2008.

Green, M. \& Oakley, B. Elite sport development systems and playing to win: uniformity and diversity in international approaches. Leis. Stud., 20(4):247-67, 2001.

Hoare, D. G. Predicting success in junior elite basketball players--the contribution of anthropometic and physiological attributes. J. Sci. Med. Sport, 3(4):391-405. 2000.
International Society for the Advancement of Kinanthropometry (ISAK). International Standards for Anthropometric Assessment. Underdale, The International Society for the Advancement of Kinanthropometry, 2006.

Jelicic, M.; Sekulic, D. \& Marinovic, M. Anthropometric characteristics of high level European junior basketball players. Coll. Antropol., 26 (Suppl.):69-76, 2002.

Norton, K. \& Olds, T. (Eds.). Antropometrica. Sidney, University of New South Wales Press, 1996.

Norton, K. \& Olds, T. Morphological evolution of athletes over the $20^{\text {th }}$ century: causes and consequences. Sports Med., 31(11):763-83, 2001.

Ochoa Martínez, P. Y.; Hall López, J. A.; Alarcón Meza, E. I.; Arráyales Millán, E. M. \& Sánchez León, R. Somatotype profile and body composition of players from the mexican professional basketball league. Int. J. Morphol., 32(3):1032-5, 2014.

Popovic, S.; Akpinar, S.; Jaksic, D.; Matic, R. \& Bjelica, D. Comparative study of anthropometric measurement and body composition between elite soccer and basketball players. Int. J. Morphol., 31(2):461-7, 2013.

Rivera-Sosa, J. M. Valoración del somatotipo y proporcionalidad de futbolistas universitarios mexicanos respecto a futbolistas profesionales. Rev. Int. Med. Cienc. Act. Fis. Deport., 6(21):16-28, 2006.

Rivera, M. A. \& Suárez, E. The somatotype of Puerto Rican male athletes at the X Pan American Games: a brief descriptive report. P. R. Health Sci. J., 9(3):207-13, 1990 .

Ross, W. D. \& Kerr, D. A. Fraccionamiento de la masa corporal: un nuevo método para utilizar en nutrición clínica y medicina deportiva. Apunts, Educ. Fís. Deport., 18:175-87, 1993.

Ross, W. D. \& Marfell-Jones, M. J. Cineantropometría. En: Mac Dougall, D.; Wegner, H. \& Green, H. (Eds.). Evaluación Fisiológica del Deportista. $3^{\mathrm{a}}$ ed. Badalona, Ed. Paidotribo S. L., 2005.

Toriola, A. L.; Salokun, S. O. \& Mathur, D. N. Somatotype characteristics of male sprinters, basketball, soccer, and field hockey players. Int. J. Sports Med., 6(6):344-6, 1985.

Valdés-Badilla, P. A.; Godoy-Cumillaf, A. E. R.; Herrera-Valenzuela, T. N. \& Ramírez-Campillo, R. Anthropometric and fitness profile of senior basketball players. Int. J. Morphol., 33(1):285-90, 2015.

Veldre, G. Heath-Carter somatotype categories and their sexual maturation differences in 12--15- year-old estonian boys and girls. Pap. Anthropol., 13:269-85, 2004.

Viviani, F. The somatotype of medium class Italian basketball players. J. Sports Med. Phys. Fitness, 34(1):70-5, 1994.

Dirección para Correspondencia:

Juan Manuel Rivera Sosa

Universidad Autónoma de Chihuahua

Facultad de Ciencias de la Cultura Física

Laboratorio para la Actividad Física y la Salud

MEXICO

Email:.jmrivera@uach.mx,jmriveras@hotmail.com

Recibido : 18-07-2015

Aceptado: $20-10-2015$ 